\title{
Raymond Hill, Terry Kenakin and Tom Blackburn (Eds): Pharmacology for Chemists-Drug Discovery in Context
}

\author{
Neil C. Henney ${ }^{1}$
}

๑) Springer-Verlag GmbH Germany, part of Springer Nature 2019

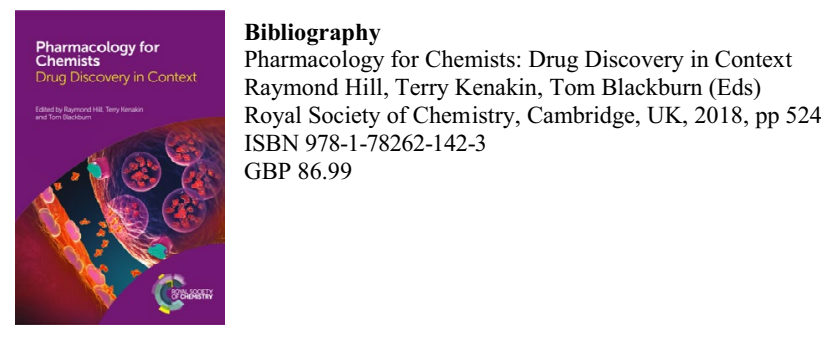

As an aid to the promotion of a more integrated and betterinformed team pursuing the goal of identifying the next big hit for pharma, Pharmacology for Chemists is a text worth reading for all budding medicinal chemists and drug discovery scientists, and perhaps even for those longer in the tooth looking for a reminder of the biological mechanisms and physiological effects which are invoked through application of the latest precipitate from the evaporating basin. Integration of understanding and cross-talk between disciplines and professions is understandably fashionable in healthcare, as well as in the drug discovery sciences, and there can be many rewards reaped from inviting those from other fields to share ideas, discuss priorities and to look at problems from a different perspective.

The fundamental principles of pharmacology can be found in innumerable textbooks and, these days, on-line ad libitum, but in this book the information is presented in an intelligent and focused manner walking the reader through a brief history of the discipline before moving on to an introduction to common pharmacological assays. The third chapter takes the reader through structure-based drug design using computational techniques to bring us up-to-date and to provide an example of the importance of cross-disciplinary understanding between chemists and pharmacologists in modern drug discovery explorations.

Neil C. Henney

N.C.Henney@ljmu.ac.uk

1 The School of Pharmacy and Biomolecular Sciences, Liverpool John Moores University, Liverpool, UK
A series of chapters on the pharmacology of drugs acting on key organs or 'body systems' then follows - these are not exhaustive as many organs and tissues are missing, but are really just examples to provide history and context to the story of the relationship between chemistry and pharmacology. Nevertheless the chapters are written well, are interesting to read and discuss many of the trials and tribulations, as well as the successes, of producing a compound that has a desirable and marketable effect. Later chapters deal with the tricky subject of what happens when promising compounds are given to humans, and how difficult it can be to predict the responses to these in real people based on pre-clinical data. These chapters are also informative and offer case studies and talk through common problems which occur in clinical trials to add further context.

Although this is a very well-constructed book, I can not help but feel that something is perhaps missing here-the push towards personalised medicine. In delivering this tailored model of healthcare, I imagine medicinal chemists might find it valuable to contextualise drug design in the new era involving an understanding of genomics as a part of the molecular basis of disease. Pharmacology for Chemists deals very well indeed with "population pharmacology", but does not venture very far into the influence of genetics on each individual's response to the same drug, and this is likely to be the key to many future drug development programmes looking for personalised approaches to managing disease.

Yet this is still a text I would thoroughly recommend to medicinal chemists, and also more broadly to other scientists involved in drug design and development, both in the pharmaceutical industry and in research institutions. A lot can be gained from better understanding of how we each contribute to programmes of research, how we each tackle problems and the barriers we face. Pharmacology for Chemists is a welcome resource to help meet this aim.

Publisher's Note Springer Nature remains neutral with regard to jurisdictional claims in published maps and institutional affiliations. 